\title{
Hypoxia-induced up-regulation of angiopoietin-2 in colorectal cancer
}

\author{
JINYU GU ${ }^{1,2}$, HIROFUMI YAMAMOTO ${ }^{1}$, MINORU OGAWA ${ }^{1}$, CHEW YEE NGAN ${ }^{1}$, KATSUKI DANNO ${ }^{1}$, \\ HIDEYUKI HEMMI $^{1}$, NAGANORI KYO ${ }^{1}$, ICHIRO TAKEMASA ${ }^{1}$, MASATAKA IKEDA ${ }^{1}$, \\ MITSUGU SEKIMOTO ${ }^{1}$ and MORITO MONDEN ${ }^{1}$
}

${ }^{1}$ Department of Surgery and Clinical Oncology, Graduate School of Medicine, Osaka University, Osaka 565-0871, Japan;
${ }^{2}$ Department of General Surgery, the Second Affiliated Hospital of Harbin Medical University, Harbin 150086, P.R. China

Received September 2, 2005; Accepted November 7, 2005

\begin{abstract}
Angiogenesis is a compensatory mechanism that enables malignant tumors to survive in an oxygen-deficient environment. To test our hypothesis that hypoxia stimulates the production of angiopoietin-2 (Ang-2) in colorectal cancer (CRC), we investigated the expression of Ang-2 in three cultured CRC cell lines, and in specimens from 11 CRC metastatic liver tumors. Hypoxia-induced Ang-2 mRNA expression was clearly evident in HCT116 cells that did not express Ang-2 under normoxic conditions. Ang-2 mRNA was detected only after $48 \mathrm{~h}$ in hypoxic serum-deprived cultures in a LoVo cell line, and under both normoxic and hypoxic conditions without any noticeable difference in the HT29 cells. There was a stepwise increase in Ang-2 expression from the periphery to the central part of the liver metastatic foci, whereas an inverse result was noted in tumor blood vessels, with a gradual decrease in CD31-positive ECs from the edge to the central region of the metastatic lesion. An expression pattern similar to Ang-2 was found in glucose transporter 1 (Glut-1), a known hypoxia-induced factor. These findings suggest that hypoxia plays an important role in inducing the expression of Ang-2 in CRC.
\end{abstract}

\section{Introduction}

It is generally accepted that CRC growth and metastasis are dependent on neovascularization, a process called 'angiogenesis' (1). The angiogenic switch involves various factors and complex processes. There is evidence that angiopoietins (Ang) are implicated in tumor angiogenesis through

Correspondence to: Dr Hirofumi Yamamoto, Department of Surgery and Clinical Oncology, Graduate School of Medicine, Osaka University, 2-2 Yamada-oka, Suita-City, Osaka 565-0871, Japan

E-mail: kobunyam@surg2.med.osaka-u.ac.jp

Key words: angiopoietin-2, hypoxia, colorectal cancer, angiogenesis a shift of balance towards Ang-2, which destabilizes the existing vasculature to a more plastic state (2).

Angiopoietins constitute a novel family of extracellular ligands that bind exclusively to the endothelium-specific tyrosine kinase receptor Tie-2 $(3,4)$. Of the four known Ang molecules (Ang-1, Ang-2, Ang-3, and Ang-4), the best characterized are Ang-1 and Ang-2. Up-regulated expression of Ang-2 has been noted in many human malignancies, including CRC (5-10).

We reported previously that high expression of Ang-2 was significantly more common in metastatic CRC than in normal mucosa and primary CRC, and the expression of Ang-2 increased from the peripheral to intermediate region of the metastatic lesion. Thus, we hypothesized that worsening hypoxia in the center of the metastatic lesion would induce overexpression of Ang-2. To our knowledge, this has not been previously investigated in $\mathrm{CRC}$, and we examined the role of hypoxia on Ang-2mRNA induction in CRC cell lines and compared its distribution with that of hypoxia, as measured by the distribution of a hypoxia marker, Glut-1, in a CRC metastatic liver tumor. We also assessed the distribution of Ang-2 and Glut-1 in relation to the supply of blood vessels, as measured by antibodies to CD31.

\section{Materials and methods}

Clinical samples. We obtained surgical specimens from 11 patients with CRC and synchronous liver metastasis. Written informed consent was obtained from all patients before the study.

Cell lines and culture conditions. The human colon cancer cell lines, HCT116, LoVo, and HT29, were obtained from the American Type Culture Collection (Manassas, VA). These cells were grown in Dulbecco's modified Eagle's medium supplemented with $10 \%$ fetal bovine serum, 100 units $/ \mathrm{ml}$ penicillin, and $100 \mu \mathrm{g} / \mathrm{ml}$ streptomycin at $37^{\circ} \mathrm{C}$ in a humidified incubator with $5 \% \mathrm{CO}_{2}$. For cultures under hypoxic conditions, $16 \mathrm{~h}$ after the last medium change, the monolayer cultures were grown for 24 or $48 \mathrm{~h}$ at $37^{\circ} \mathrm{C}$ in a continuously monitored atmosphere of $1 \% \mathrm{O}_{2}, 5 \% \mathrm{CO}_{2}$, and $94 \% \mathrm{~N}_{2}$ gas mixture using a multigas incubator, model 9200 (Wakenyaku 
Table I. Primers used in the RT-PCR assay.

\begin{tabular}{lllr}
\hline Gene & Sense primer & Antisense primer & $\begin{array}{r}\text { Product } \\
\text { size (bp) }\end{array}$ \\
\hline Ang-2 & 5'-GACGGCTGTGATGATAGAAATAGG-3' & 5'-GACTGTAGTTGGATGATGTGCTTC-3' & 264 \\
Glut-1 & 5'-CGGGCCAAGAGTGTGCTAAA-3' & 5'-TGACGATACCGGAGCCAATG-3' & 278 \\
ß-actin & 5'-GAAAATCTGGCACCACACCTT-3' & 5'-GTTGAAGGTAGTTTCGTGGAT-3' & 590 \\
\hline
\end{tabular}

Table II. RT-PCR assay conditions.

\begin{tabular}{|c|c|c|c|c|c|}
\hline Gene & $\begin{array}{l}\text { Amount of } \\
\text { each primer } \\
\text { used (pmol) }\end{array}$ & $\begin{array}{l}\beta \text {-actin } \\
(\mathrm{pmol})\end{array}$ & Denaturing cycle & PCR cycles & $\begin{array}{l}\text { Extension } \\
\text { cycle }\end{array}$ \\
\hline Ang-2 & 5 & 1 & $95^{\circ} \mathrm{C}, 12 \mathrm{~min}$ & $\begin{array}{l}95^{\circ} \mathrm{C}, 1 \mathrm{~min} ; \\
53^{\circ} \mathrm{C}, 1 \mathrm{~min} ; \\
72^{\circ} \mathrm{C}, 1 \mathrm{~min} \\
\times 35 \text { cycles }\end{array}$ & $72^{\circ} \mathrm{C}, 10 \mathrm{~min}$ \\
\hline Glut-1 & 5 & 1 & $95^{\circ} \mathrm{C}, 12 \mathrm{~min}$ & $\begin{array}{l}95^{\circ} \mathrm{C}, 1 \mathrm{~min} ; \\
58^{\circ} \mathrm{C}, 1 \mathrm{~min} ; \\
72^{\circ} \mathrm{C}, 1 \mathrm{~min} ; \\
\times 35 \text { cycles }\end{array}$ & $72^{\circ} \mathrm{C}, 10 \mathrm{~min}$ \\
\hline
\end{tabular}

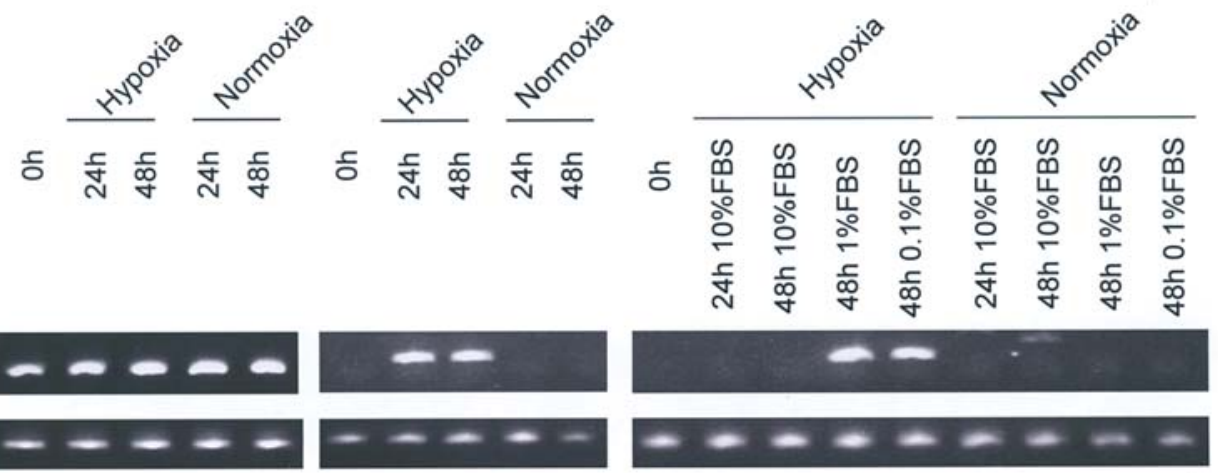

Figure 1. Induction of angiopoietin-2 (Ang-2) mRNA expression by hypoxia in colon cancer cell lines. In the HT29 cell line, Ang-2 mRNA was detected under both normoxic and hypoxic conditions without a noticeable difference. In the HCT116 cell line, Ang-2 mRNA was not detected under normoxic conditions, but was clearly found after 24 and $48 \mathrm{~h}$ under hypoxic conditions. In the LoVo cell line, Ang- 2 mRNA expression was observed only after $48 \mathrm{~h}$ under hypoxic conditions in hypoxic serum-deprived cultures, supplemented with $1.0 \%$ or $0.1 \%$ FBS, but not $10 \%$ FBS.

Co., Kyoto, Japan). Cultures were prepared in normoxia as a control reference.

Laser capture microdissection (LCM). LCM was performed in frozen tissue samples using the LM200 LCM system (Arcturus Engineering, Santa Clara, CA) as described previously (10).

RNA extraction and semi-quantitative duplex RT-PCR. Total RNA was extracted from colon cancer cells and clinical samples by a single-step method using Trizol reagent (Life Technologies, Gaithersburg, MD). Complementary DNA (cDNA) was generated using avian myeloblastosis virus reverse transcriptase (Promega, Madison, WI). Semiquantitative analyses for the expression of Ang-2 and Glut-1 mRNA were performed by the duplex RT-PCR techniques, as described previously (10). We used $B$-actin as the internal standard. The PCR primer sequences and conditions are shown in Tables I and II.

Reagents and immunohistochemical assay. The anti-human antibodies used in this study were goat polyclonal Ang-2 (Santa Cruz Biotecnology), rabbit polyclonal Glut-1 (Dako, Carpinteria, CA), and mouse monoclonal CD31 (Dako). Immunohistochemical assay of Ang-2, Glut-1 and CD31 was performed using the Vectastain avidin-biotin complex peroxidase kit (Vector Laboratories, Burlingame, CA), as described previously (10). 


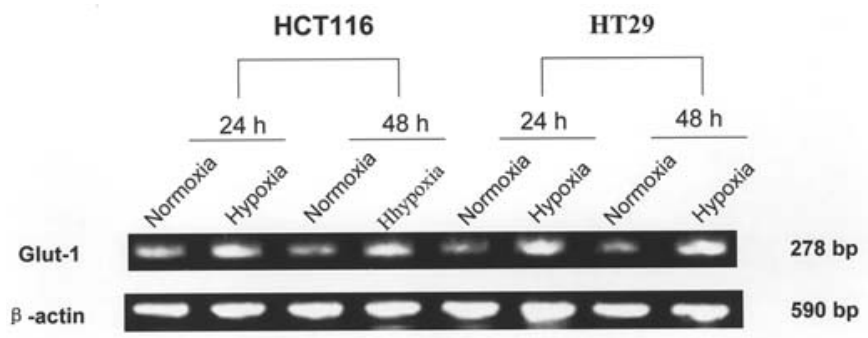

Figure 2. Hypoxia enhanced the expression of Glut-1 mRNA in colorectal cancer cell lines. In the HCT116 and HT29 cancer cell lines, hypoxia clearly enhanced the Glut-1 mRNA level after 24 and $48 \mathrm{~h}$ compared with the level under normoxic conditions.

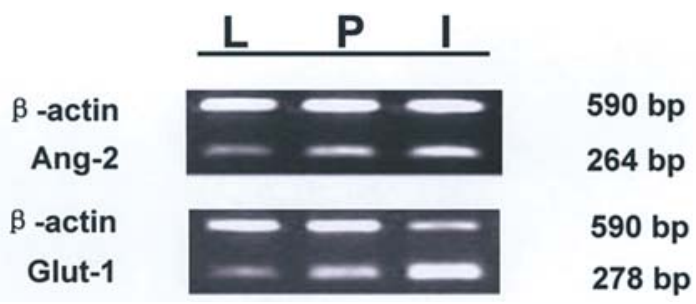

Figure 3. Expression of Ang-2 mRNA and Glut-1 mRNA in the metastatic liver tumors. Higher expression of Ang-2 mRNA was found in the metastatic liver tumor, especially in the intermediate region, compared to adjacent liver tissue. The expression patterns of Glut-1 were similar to those of Ang-2 in each area.

Statistical analysis. Statistical analysis was performed using StatView J-5.0 software (Abacus Concepts, Berkeley, CA). Medians were compared using the Wilcoxon signed-rank test, and $\mathrm{p}<0.05$ was accepted as significant.

\section{Results}

Induction of Ang-2 and Glut-1 expression by hypoxia in the colorectal cancer cell lines. RT-PCR analyses did not detect Ang-2 mRNA in the HCT116 or LoVo cell lines under normoxic conditions. Ang-2 mRNA was detected in the HT29 cell line, under normoxic and hypoxic conditions without any noticeable difference. Hypoxia-induced Ang-2 mRNA expression was observed in the HCT116 cell line, whereas in the LoVo cell line, it was only induced after $48 \mathrm{~h}$ in hypoxic serum-deprived cultures supplemented with $1 \%$ or $0.1 \%$ FBS, but not $10 \%$ FBS (Fig. 1). The culture of HCT116 and HT29 cells enhanced the expression of Glut-1 mRNA after 24 and $48 \mathrm{~h}$ under hypoxic conditions, but not under normoxic conditions (Fig. 2).These experiments were repeated 3 times with reproducible results.

Expression of Ang-2 $m R N A$ and Glut-1 mRNA in the liver metastatic tumors. We measured the levels of Ang-2 mRNA and Glut-1 mRNA by semi-quantitative RT-PCR after LCM. Ang-2 mRNA expression was detected in all 11 liver metastatic lesions, being stronger within the metastatic lesion than in bordering liver tissue (Fig. 3). The ranking intensity of Ang-2 mRNA expression was located in the intermediate area of the metastasis. We found a similar expression pattern in Glut-1 mRNA (Fig. 4A). A stepwise increase in Ang-2 and Glut-1 expression from the adjacent liver to the periphery, then to the intermediate area of the metastatic tumors with
A

Ang-2

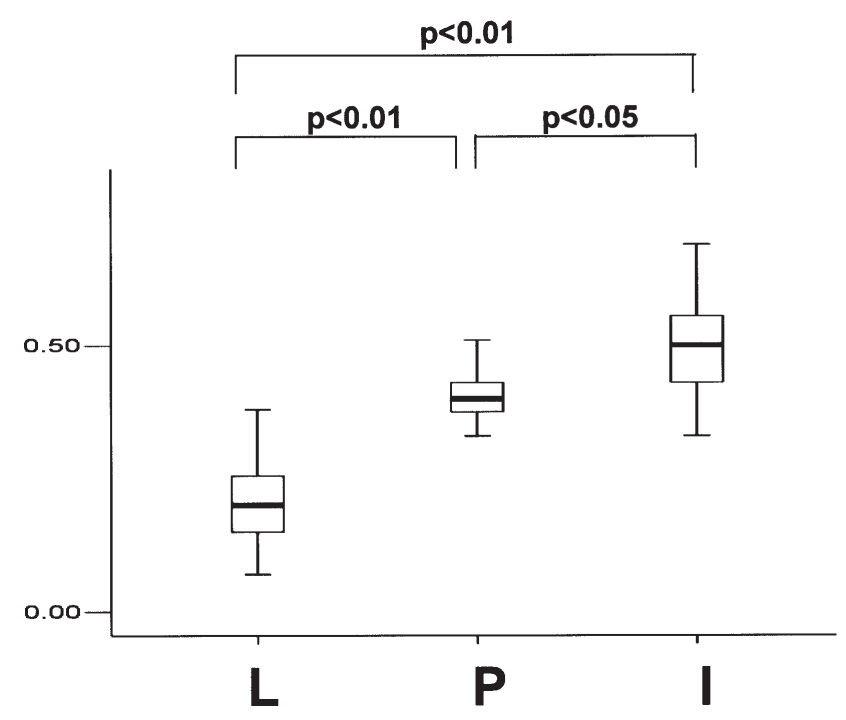

B

\section{Glut-1}

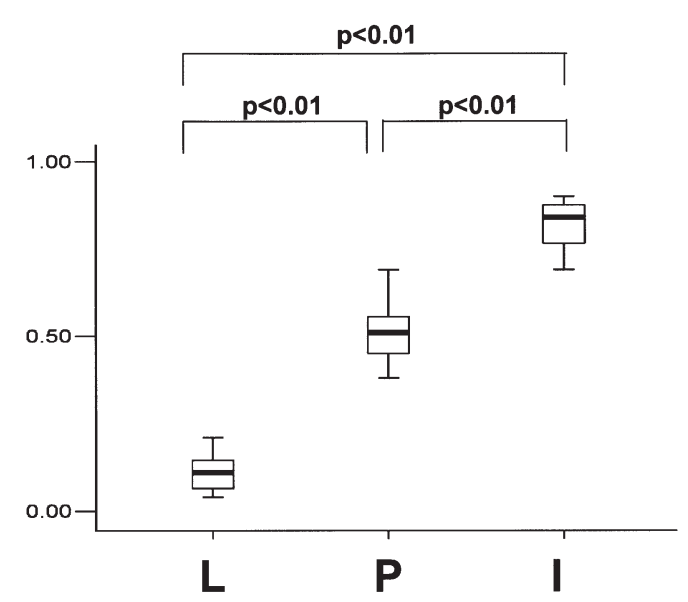

Figure 4. Quantity of Ang-2 and Glut-1 in each region. The expression of each molecule was normalized by B-actin. (A) The median values of Ang-2 were: liver (L), 0.2; periphery (P), 0.4; and intermediate, 0.5. (B) The median values of Glut-1 were: liver $(\mathrm{L}), 0.11$; periphery $(\mathrm{P}), 0.51$; and intermediate, 0.84 . The Wilcoxon signed-rank test indicated a significant stepwise increase in Ang-2 and Glut-1 in each pair of adjacent regions.

increased expression of Ang-2 and Glut-1, was normalized by $\beta$-actin (Fig. 4B).

Immunohistochemical findings in the liver metastatic tumors. We observed an increase in the expression of Ang-2 protein from the periphery to intermediate region of the metastatic tumor (Fig. 5). A similar pattern of staining was seen in Glut-1, whereas an inverse result was noted in CD31-positive ECs, with a gradual decrease from the edge to the central region of the metastatic lesion.

\section{Discussion}

Our data support the hypothesis that hypoxia induces Ang-2 expression in CRC. Immunohistochemical (IHC) assay showed low vascular density and high Ang-2 expression in the intermediate part of the metastatic lesion near the central area 

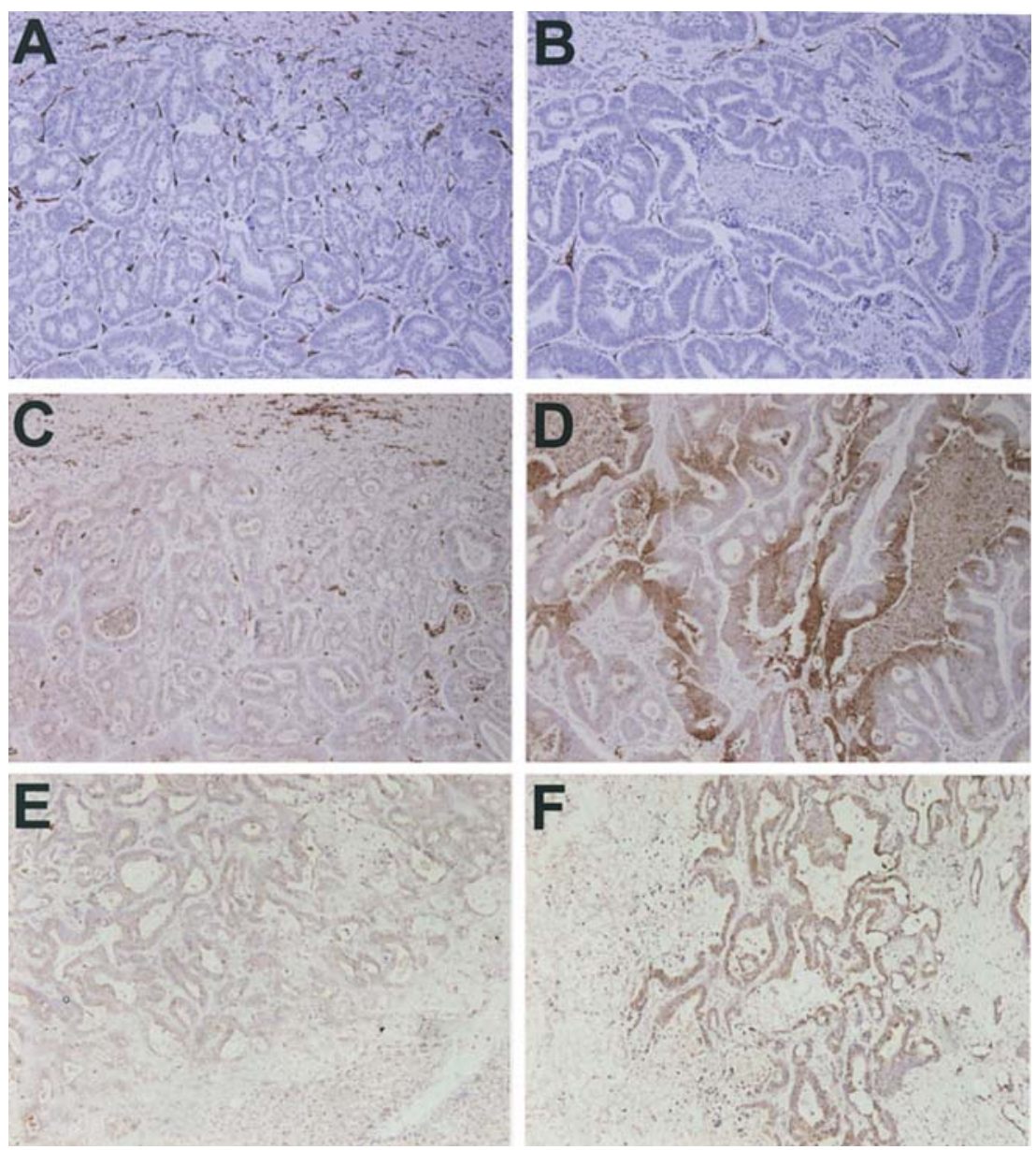

Figure 5. Immunohistochemical analyses of CD31, Glut-1 and Ang-2 in different regions of the metastatic liver tumor. (A) A CD31-stained section from the peripheral part of the metastatic liver tumor. (B) A CD31-stained section from the intermediate part of the metastatic liver tumor, where vessels were not as abundant as in the peripheral part. (C) An angiopoietin-2 (Ang-2)-stained section from the peripheral part of the metastatic liver tumor. (D) An Ang-2-stained section from the intermediate part of the metastatic liver tumor, where tumor cells, particularly around the necrosis, showed a higher intensity of Ang- 2 staining than in the peripheral region. (E) A Glut-1-stained section from the peripheral part of the metastatic liver tumor. (F) A Glut-1-stained section from the intermediate part of the metastatic liver tumor. Glut-1 also showed stronger intensity in the intermediate part of the metastatic liver tumor (original magnification, $\mathrm{x} 100)$.

of necrosis, in accordance with our previous study (10). Considering the similar expression patterns of Glut-1 and the role of Ang-2 in tumor angiogenesis, it is reasonable to attribute this phenomenon to tissue hypoxia, an environmental factor relating to several tumor biological characteristics including angiogenesis. The presence of necrosis within tumors is thought to be a marker of hypoxia (11) and Glut-1 is well validated as a predictor of hypoxia in CRC (12). In tumor angiogenesis, Ang-2 acts as a destabilizing signal, expressed at the sites of vessel sprouting and regression (13). Thus, we assume that as the tumor enlarges, the hypoxia inside the tumor becomes more severe, which in turn stimulates tumor cells exposed to the most hypoxic conditions to produce and secrete Ang-2.

To ameliorate the hypoxic conditions, the hypoxia-driven Ang-2 induced the ECs of surrounding blood vessels to detach from the pericytes and basement membrane and migrate to the peripheral part of the tumor, resulting in neovascularization in undervascularized areas. Ang-2 was found to stimulate the migration and tubular formation of mouse brain capillary ECs (14). Although it is widely accepted that most tumors and metastases originate as an avascular mass, evidence suggests that tumors in more natural settings may initially grow by co-opting existing host vessels, particularly when they arise within or metastasize to vascularized tissue (15). These co-opted vessels regress, but the remaining tumor is rescued by angiogenesis at its margin. The overexpression of Ang-2 may be a critical regulator in vascular regression (13).

RT-PCR clearly showed that hypoxia is capable of inducing Ang-2 expression in HCT116 CRC cell lines. Hypoxicinduced Ang-2 expression has also been reported in epithelial cells and glioma cells $(5,16,17)$. Unlike HCT116 cells and human glioma cells, in which hypoxic stimulation directly induces Ang-2 (18), a decrease in both the oxygen concentration and the nutrient supply induced Ang-2 expression in the LoVo CRC cell line, which suggests that poor nutrition may be another stimulus of Ang-2 induction. The action of Ang was also found to be cell-specific. Ahmad et al reported that 14 of 18 colon carcinoma cell lines expressed Ang-2, which may explain the expression pattern of Ang-2 in HT29 cells (9). Moreover, the effect of hypoxia on Ang-2 expression varies in different tumor cells. Hypoxic stimuli were not responsible for Ang-2 up-regulation in human hepatocellular carcinoma cells, and down-regulation was seen in renal cell carcinoma cells $(19,20)$. 
In summary, the findings of this study suggest that the expression of Ang-2 in CRC is a complex mechanism; however, hypoxia plays an important role in its induction. Moreover, the insufficient blood supply and resultant hypoxia in liver metastatic foci, especially in the intermediate region, might stimulate CRC cells to produce Ang-2.

\section{Acknowledgements}

This work was supported by a Grant-in-Aid for Cancer Research from the Ministry of Education, Science, Sports, and Culture Technology, Japan (to H.Y.). This study was also supported in part by a Japan-China Sasakawa Medical Fellowship (to Jinyu Gu).

\section{References}

1. Ellis LM: Angiogenesis and its role in colorectal tumor and metastasis formation. Semin Oncol 31: 3-9, 2004.

2. Tait CR and Jones PF: Angiopoietins in tumors: the angiogenic switch. J Pathol 204: 1-10, 2004.

3. Davis S, Aldrich TH, Jones PF, Acheson A, Compton DL, Jain V, Ryan TE, Bruno J, Radziejewski C, Maisonpierre PC and Yancopoulos GD: Isolation of angiopoietin-1, a ligand for the TIE2 receptor, by secretion-trap expression cloning. Cell 87: 1161-1169, 1996.

4. Maisonpierre PC, Suri C, Jones PF, Bartunkova S, Wiegand SJ, Radziejewski C,Compton D, McClain J, Aldrich TH, Daly TJ, Papadopoulos N, Davis S, Sato TN and Yancopoulos GD: Angiopoietin-2, a natural antagonist for Tie2 that disrupts in vivo angiogenesis. Science 277: 55-60, 1997.

5. Koga K, Todaka T, Morioka M, Hamada J, Kai Y, Yano S, Okamura A, Takakura N, Suda T and Ushio Y: Expression of angiopoietin-2 in human glioma cells and its role for angiogenesis. Cancer Res 61: 6248-6254, 2001.

6. Sfiligoi C, de Luca A, Cascone I, Sorbello V, Fuso L, Ponzone R, Biglia N, Audero E, Arisio R, Bussolino F, Sismondi P and De Bortoli M: Angiopoietin-2 expression in breast cancer correlates with lymph node invasion and short survival. Int $\mathbf{J}$ Cancer 103: 466-474, 2003.

7. Caine GJ, Blann AD, Stonelake PS, Ryan P and Lip GY: Plasma angiopoietin-1, angiopoietin-2 and Tie-2 in breast and prostate cancer: a comparison with VEGF and Flt-1. Eur J Clin Invest 33: 883-890, 2003.

8. Yoshida Y, Oshika Y, Fukushima Y, Tokunaga T, Hatanaka H, Kijima H, Yamazaki H, Ueyama Y, Tamaoki N, Miura S and Nakamura M: Expression of angiostatic factors in colorectal cancer. Int J Oncol 15: 1221-1225, 1999.
9. Ahmad SA, Liu W, Jung YD, Fan F, Reinmuth N, Bucana CD and Ellis LM: Differential expression of angiopoietin-1 and angiopoietin-2 in colon carcinoma. A possible mechanism for the initiation of angiogenesis. Cancer 92: 1138-1143, 2001.

10. Ogawa M, Yamamoto H, Nagano H, Miyake Y, Sugita Y, Hata T, Kim BN, Ngan CY, Damdinsuren B, Ikenaga M, Ikeda M, Ohue M, Nakamori S, Sekimoto M, Sakon M, Matsuura N and Monden M: Hepatic expression of ANG2 RNA in metastatic colorectal cancer. Hepatology 39: 528-539, 2004.

11. Koch CJ, Chasan JE, Jenkins WT, Chan CY, Laughlin KM and Evans SM: Co-localization of hypoxia and apoptosis in irradiated and untreated HCT116 human colon carcinoma xenografts. Adv Exp Med Biol 454: 611-618, 1998.

12. Cooper R, Sarioglu S, Sokmen S, Fuzun M, Kupelioglu A, Valentine H, Gorken IB, Airley R and West C: Glucose transporter-1 (Glut-1): a potential marker of prognosis in rectal carcinoma? Br J Cancer 89: 870-876, 2003.

13. Holash J, Maisonpierre PC, Compton D, Boland P, Alexander CR, Zagzag D, Yancopoulos GD and Wiegand SJ: Vessel co-option, regression, and growth in tumors mediated by angio-poietins and VEGF. Science 284: 1994-1998, 1999.

14. Mochizuki Y, Nakamura T, Kanetake $H$ and Kanda S: Angiopoietin 2 stimulates migration and tube-like structure formation of murine brain capillary endothelial cells through c-Fes and c-Fyn. J Cell Sci 115: 175-183, 2002.

15. Pezzella F, Pastorino U, Tagliabue E, Andreola S, Sozzi G, Gasparini G, Menard S, Gatter KC, Harris AL, Fox S, Buyse M, Pilotti S, Pierotti M and Rilke F: Non-small-cell lung carcinoma tumor growth without morphological evidence of neoangiogenesis. Am J Pathol 151: 1417-1423, 1997.

16. Krikun G, Schatz F, Finlay T, Kadner S, Mesia A, Gerrets R and Lockwood CJ: Expression of angiopoietin-2 by human endometrial endothelial cells: regulation by hypoxia and inflammation. Biochem Biophys Res Commun 275: 159-163, 2000.

17. Oh H, Takagi H, Suzuma K, Otani A, Matsumura M and Honda Y: Hypoxia and vascular endothelial growth factor selectively upregulate angiopoietin-2 in bovine microvascular endothelial cells. J Biol Chem 274: 15732-15739, 1999.

18. Hartmann A, Kunz M, Kostlin S, Gillitzer R, Toksoy A, Brocker EB and Klein CE: Hypoxia-induced up-regulation of angiogenin in human malignant melanoma. Cancer Res 59: 1578-1583, 1999.

19. Sugimachi K, Tanaka S, Taguchi K, Aishima S, Shimada M and Tsuneyoshi M: Angiopoietin switching regulates angiogenesis and progression of human hepatocellular carcinoma. J Clin Pathol 56: 854-860, 2003.

20. Currie MJ, Gunningham SP, Turner K, Han C, Scott PA, Robinson BA, Chong W, Harris AL and Fox SB: Expression of the angiopoietins and their receptor Tie 2 in human renal clear cell carcinomas; regulation by the von Hippel-Lindau gene and hypoxia. J Pathol 198: 502-510, 2002. 